\title{
A Routing Algorithm for Wireless Sensor Networks based on Vector Field Theory
}

\author{
http://dx.doi.org/10.3991/ijoe.v12i03.5407 \\ Ming Li ${ }^{1,2}$, Huan Yan Qian ${ }^{1}$ \\ ${ }^{1}$ Nanjing University of Science and Technology, Nanjing, China \\ ${ }^{2}$ Hohai University, Nanjing, China
}

\begin{abstract}
To standardize network load and increase throughput, a new scheme is proposed to analyze the routing of wireless sensor networks (WSNs). First, the WSN model was established based on field theory with vectors and operators to analyze the transfer process of message packets given that the source node in such networks is characterized by source points while the sink node can be regarded as a gathering point. Second, a vector routing algorithm was developed for a WSN that was modeled according to field theory. Finally, the WSN routing algorithm was simulated in given two scenarios; simulation results show that throughput can be significantly increased by choosing routes such that traffic is spread as uniformly as possible throughout the network.
\end{abstract}

Index Terms - Load Balancing, Multipath Routing, Vector Field Theory, Wireless Sensor Network.

\section{INTRODUCTION}

A wireless sensor network (WSN) is a typical nodeintensive network. Given the scale and complication of network node deployment and the asymmetry of network flow, load balancing has become a characteristic to be emphasized in research on WSN routing [1][2]. The present study aims to determine the multipath routing process that standardizes network loads as much as possible to reduce the chance of collision and to increase throughput [3].

The typical methods used in WSN routing research are the link state and the distance vector algorithms [4][5][6]. Fisheye state routing [7] and the ad hoc on-demand distance vector (AODV) [8][9] are two WSN protocols. Other previous studies provide detail on WSN routing protocols [10][11].

The current work first examines the static WSN as the research object; the source-destination node pairs and node position information are known in this case. Then, the study assumes that the center router can compute routing information for different source-destination nodes. The hypothesis is reasonable for WSNs, although it is too strict for other applications. Moreover, this inference can be used to initialize static routing. This research assumes that the constraint can be removed in follow-up work. For instance, related information such as the positions of source and destination nodes can be informed through the flooding mechanism, which aims to facilitate the distributed computation of routing information.

When communication with other nodes is necessary, the source node can be considered the load source. Communication is realized in virtue of transmission media due to the spatial distance between the source and destination nodes. In general, intermediate nodes should have forwarding capabilities; thus, the path between the source and destination nodes contains intermediate nodes. The future objective of this research is to define the load function of space rather than the node function.

\section{MAthematical Model of A Vector FiEld}

This study constructs a WSN containing $\mathrm{N}$ nodes that can communicate with one another. The nodes are distributed in area A randomly. Under the assumption that $\mathrm{M}$ source-destination node pairs $\mathrm{s}_{1}, \mathrm{~s}_{2}, \ldots, \mathrm{s}_{\mathrm{m}}$ are present, $\mathrm{W}_{\mathrm{i}}$ represents the bandwidth requirement (weight) of node pair $\mathrm{s}_{\mathrm{i}}$. Presumably, $\mathrm{S}_{\mathrm{i}}$ chooses one or multiple paths in the area and each path starts from the source node of $s_{i}$ and ends at the corresponding destination node. Thus, the value of $\mathrm{W}_{\mathrm{i}}$ is related to corresponding paths, and the weight assigned to each path represents the resource demand of the path. For clarity, the chosen path is not restricted to the position of the intermediate node; rather, the path is used to represent the "abstract" concept of a message transmission line. Once communication has been initiated among nodes, each abstract path must be transformed into an actual route that connects the source and destination nodes. This route may pass several intermediate nodes.

A vector field in area A [12] is defined, the weight of each source-destination node path is known, and $\vec{D}$ represents the load vector field of the network. Presumably, $(x, y) \in A$, and a minimum area unit $\mathrm{S}$ near point $(\mathrm{x}, \mathrm{y})$ is selected. For each path passing through $\mathrm{S}$, the direction and size of its tangent vector are defined, as shown in (1). Mathematically, if the number of sensors is limited, then the value of $\vec{D}$ will be 0 only if the collection is empty.

$$
\vec{D}=\lim _{|S| \rightarrow 0} \frac{1}{|S|} \sum_{L_{i} \cap S \neq \varnothing} W_{i} \hat{l}_{i} .
$$

where $S$ represents an area in the network and $L_{i}$ represents the path of the $i^{\text {th }}$ source-destination node pair. $\hat{l}_{i}$ represents the unit tangent vector of path $L_{i}$ and points to the destination node in the direction of the path, as shown in Fig. 1. Equation (1) shows that $\vec{D}$ is the position function. 


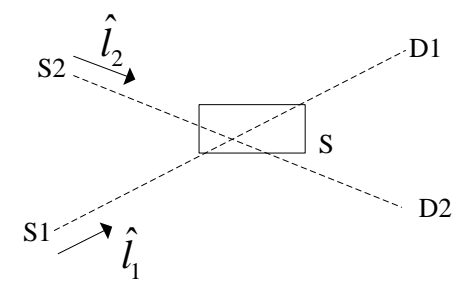

Figure 1. Path-based Load Vector

The $\vec{D}$ defined in (1) satisfies (2):

$$
\int_{C} \vec{D} \overrightarrow{d_{n}}=w
$$

where $C$ represents a closed curve, $\overrightarrow{d_{n}}$ denotes the normal differential vector of each point in the closed curve, $\sqcup$ indicates the inner product of two vectors, and $w$ is the total load of internal nodes in the closed area. To calculate the value of $w$, the weight of the source node is assigned a positive sign, and the weight of the destination node is negative. Equation (2) is similar to the Gauss law in electrostatic theory. Interestingly, the formula is similar to the form of Gauss law in the electrostatic field, which indicates that the electric flux passing through a closed curved surface is directly proportional to the quantity of electric charges enclosed by the closed curved surface.

$\rho$ represents the characteristic function of a message entering the plane domain, and its value is the position function value, as shown in (3):

$$
\rho=\lim _{|S| \rightarrow 0} \frac{1}{|S|} \sum_{u_{i} \in S} \operatorname{sgn}\left(u_{i}\right) W_{i} .
$$

where $u_{i}$ represents the mark of the source or the destination node under the weight of $W_{i}$. If $u_{i}$ denotes the source node, then $\operatorname{sgn}\left(u_{i}\right)=1$; otherwise, $\operatorname{sgn}\left(u_{i}\right)=-1$. According to this definition, Equation (2) can be rewritten in the form of a difference equation:

$$
\begin{aligned}
& \vec{\Delta} \cdot \vec{D}=\rho \\
& \vec{\Delta}=\frac{\partial}{\partial_{x}} \hat{i}+\frac{\partial}{\partial_{x}} \hat{j}
\end{aligned}
$$

where $x$ and $y$ represent the horizontal and vertical coordinates in the Cartesian rectangular coordinate system, respectively, and $\hat{i}$ and $\hat{j}$ denote the unit normal vectors along the $x$-axis and $y$-axis coordinates, respectively.

Mathematically, if the number of source-destination node pairs is limited, then the values of $\vec{D}$ and $\rho$ as defined in (1) and (3) are 0 only if the collection is empty. In fact, the number of source-destination node pairs is not large; therefore, $S$ is adequately small. The network area can be divided into several rectangular regions by horizontal and vertical grid lines. When the rectangular units are small enough, $\vec{D}$ can be handled as a continuous variable.

$\vec{D}$ may not reflect the actual communication demand of a network in special cases. The following scene is considered: presumably, two source-destination node pairs are situated in opposite positions with mutually counteracted loads, as shown in Fig. 2. Therefore, source- destination node pairs can be divided into groups with mutually counteracted pairs that are included in different groups such that each group has a load vector field.

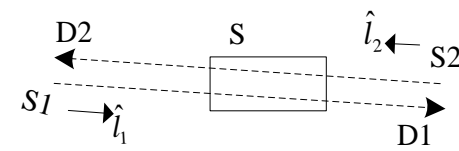

Figure 2. $\vec{D}$ that Does Not Represent the Actual Network Load in the Mutually Counteracted Case

\section{LOAD BALANCING AND ROUTING}

A collection of source-destination node pairs is given, and then the load vector field $\vec{D}$ is determined according to the aforementioned definition. A line connection is assumed between the source and the destination nodes. In this case, $\vec{D}$ satisfies the following equation:

$$
\left\{\begin{array}{l}
\vec{\nabla} \cdot \vec{D}=\rho \\
D_{n}(\mathrm{z})=0, \mathrm{z} \in \text { the edge of area A }
\end{array} .\right.
$$

In the equations, A represents the area collection of the network and $D_{n}(z)$ represents the scalar of $\vec{D}$ along area A. The first equation in (5) shows that the messages generated in the network all flow to the sink node eventually; the second equation originates from the fact that no message traffic flows out of the boundaries of the network area.

Intuitively, Equation (5) is valid for any load vector field. In fact, the load vector field is also defined as the vector field that satisfies (5). The unique $\vec{D}$ value is determined with the aforementioned equation, and then the routing is inferred. The current work first analyzes the case of multisource and single-sink nodes; subsequently, the conclusion drawn is applied to the case of multisource and multi-sink nodes (any two nodes can communicate with each other).

\section{A. Multisource and Single-sink Vector Field Routing}

The multisource and single-sink network has only one destination node and several source nodes. A large network is generally divided into several clusters; nodes must send messages to the cluster-head node to communicate with other nodes. The communication between cluster nodes and the cluster head can be considered a multisource and single-sink scenario.

In this situation, the route to the destination node can be determined by generating a $\vec{D}$ value that satisfies (5). To define the route based on $\vec{D}$, this paper proposes the concept of load line, which is similar to the electric field line in an electrostatic field and the flow line in a flow field. The load line is a transient smooth curve in the load vector field where message units are transmitted in a direction that coincides with the tangential direction of the point. The load line is clearly similar to the electric field and the flow lines, with the exception of a few special points that can neither turn nor intersect. The flow field lines between the source and the destination nodes do not intersect; in case of intersection, the value is not unique. The other property of a load line is that its direction is pointed from the source node to the destination node. 
According to the load line concept, the source node sends messages in the direction of the load line within the transmission range. As shown in Fig. 3, all nodes are presumably distributed densely in the network, and the connection line between $\mathrm{Si}$ and R1 is close to the load line. $\mathrm{R} 1$ forwards the messages of Si to R2, which is also in the direction of the load line. This line always ends at the destination node, and factually, messages from the source node can be sent to the destination node along the load line. In Fig. 3, the arrows indicate the direction of the load line; this line is similar to the wireless link between nodes.

In theory, the following case may suggest that an infinite load line starts from different source nodes, and the problem to be solved involves selecting a load line to determine the route [13][14][15]. $\mathrm{P}$ paths are assumed to be established between the source and the destination nodes, and these paths are uniformly divided from the source node. For instance, if $\mathrm{P}=8$, then the routing of each source node is uniformly distributed across eight paths, i.e., eight routes run from the source to the destination nodes, and the angle between the path and the load line in a source node is $360 \% / 8=45^{\circ}$. The number of messages transmitted in each path is proportional to the size of the integral of $\vec{D}$ along the load line in the path. The source node alone can "split," and with this "split," messages are transmitted to the destination node in the optimum $\vec{D}$ direction. In the multisource and single-sink scenario, only one sink node exists; thus, no mutually counteracting problem is illustrated in Fig. 2.

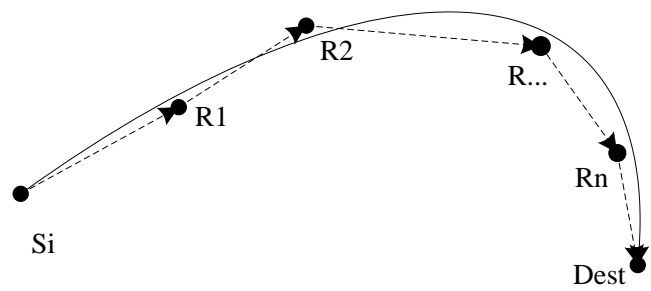

Figure 3. Selection of a Transmission Path with the Load Line

Thus far, this research has established the concept of vector fields and described the connection between the load vector field and the multisource and single-sink network routing, i.e., that routing information can be obtained with a given $\vec{D}$ value. However, Equation (5) does not generate a unique $\vec{D}$; therefore, the present problem involves increasing the restricted conditions to determine this value. An intuitive method involves constructing a uniform $\vec{D}$, and the route obtained in this manner can cause network messages to be highly scattered, thus reducing node congestion and conflict as well as enhancing throughput.

The load balancing problem can be transformed into the problem of obtaining the minimum value for the following equation:

$$
J(\vec{D})=\int_{A}\left|\left(\vec{D}-\overrightarrow{X_{a v}}\right)\right|^{2} d s .
$$

where $\overrightarrow{X_{a v}}$ is the mean value of vector $\vec{X}$ in collection A. This variable is defined as follows:

$$
\overrightarrow{X_{a v}}=\frac{1}{|A|} \int_{A} \vec{X} d s \text {. }
$$

The load is distributed as uniformly as possible because of the quadratic in (6), which prevents some areas from overloading and others from insufficient resource utilization. Interestingly, this function is similar to the definition of energy in magnetic field theory. The aforementioned problem can be summarized as follows:

$$
\left\{\begin{array}{l}
\min \text { imize } J(\vec{D})=\int_{A}\left|\left(\vec{D}-\overrightarrow{X_{a v}}\right)\right|^{2} d s \\
\vec{\nabla} \cdot \vec{D}=\rho \\
D_{n}(\mathrm{z})=0, \mathrm{z} \in \text { the edge of area } \mathrm{A}
\end{array} .\right.
$$

The optimization problem in (8) should be solved based on the following theorem:

Theorem: If $\overrightarrow{D^{*}}$ is the optimum solution of (8), then the following equation is satisfied:

$$
\vec{\nabla} \times \overrightarrow{\mathrm{D}^{*}}=0
$$

$\vec{\nabla} \times$ is a $2 \mathrm{D}$ operation under the following operation rule:

$$
\vec{\nabla} \times \vec{F}=\left(-\frac{\partial_{F_{x}}}{\partial_{y}}+\frac{\partial_{F_{y}}}{\partial_{x}}\right) \hat{k}
$$

where $\hat{k}$ is the unit vector perpendicular to $\hat{i}$ and $\hat{j}$ and $\hat{k}=\hat{i} \times \hat{j}$. Ref. [13] provides the proof of the theorem. According to this theorem, the following equation is generated:

$$
\vec{\nabla} \overrightarrow{\mathrm{D}^{*}}=\rho \vec{\nabla} \times \overrightarrow{\mathrm{D}^{*}}=0 .
$$

The aforementioned formula is similar to Maxwell's equations in electrostatic field theory. According to partial differential equation theory, the formula above can help determine the unique $\overrightarrow{D^{*}}$ value when combined with (6). Interestingly, Maxwell's equation in electrostatic field theory suggests that the space storing energy is the smallest, but in this study, the definition of energy differs from that presented under electrostatic field theory.

Mathematically, the vector field that satisfies $\vec{\nabla} \times \overrightarrow{\mathrm{D}^{*}}=0$ is called the conservative vector field. As shown in (12),

$$
\vec{D}=\vec{\nabla} U
$$

where $U$ is the gradient function. Equation (11) can be redefined as follows:

$$
\nabla^{2} U=\rho
$$

where $\nabla^{2}$ is defined as follows:

$$
\nabla^{2}=\frac{\partial^{2}}{\partial_{x}^{2}}+\frac{\partial^{2}}{\partial_{y}^{2}} .
$$

The gradient of $U$ in boundaries is 0 , i.e.,

$$
\vec{\nabla} U(\mathrm{z}) \cdot \hat{n}(\mathrm{z})=0 \quad \mathrm{z} \in \text { the edge of area } \mathrm{A}
$$

where $\hat{n}(z)$ is the unit vector pointing to the boundaries. 
The partial differential equation defined by (13) is a Poisson's equation that can effectively explain the multisource and single-sink scenario. First, the function indicates the general energy consumption for the transmission of messages from the source node to the destination node. Different source and destination nodes have varying energy consumption levels. Subsequently, the function is related to routing. Equation (13) shows that routes may be generated in the gradient direction of a potential function. This function is generated monotonously in the path of source and destination nodes, thus avoiding problems such as routing loop.

\section{B. Multisource and Multi-sink Vector Field Routing}

This work extends the existing research to a general case, i.e., any pair of nodes can communicate with each other. This case can be divided into many multisource and single-sink scenarios. The situation involving many destination nodes and the need for all source nodes to communicate with these destination nodes is considered; $\mathrm{m}$ destination nodes are presumably marked as $\Omega=\left\{d_{1}, d_{2}, \ldots, d_{m}\right\}$ in turn.

This paper discusses the uniform utilization of resources in this general case. $\overrightarrow{D_{i}}$ and $\rho_{i}$ represent the load vector field and the characteristic function in the situation involving the source node and the $i^{\text {th }}$ destination node, respectively:

$$
\vec{\nabla} \cdot \overrightarrow{D_{i}}=\rho_{i} .
$$

where $\vec{D}=\sum_{i=1}^{\mathrm{m}} \overrightarrow{D_{i}}$.

To fully define $\vec{D}$, the conditions satisfying the optimum solution must be determined. The following equation set is a method to solve load balancing:

$$
\left\{\begin{array}{l}
\min \text { imize } J=\sum_{i=1}^{m} \int_{A}\left|\left(\bar{D}_{i}-\vec{D}_{\text {iav }}\right)\right|^{2} d s \\
\vec{\nabla} \cdot \vec{D}_{i}=\rho_{i} \quad 1 \leq i \leq m \\
D_{n i}(\mathrm{z})=0, \mathrm{z} \in \text { the edge of area } \mathrm{A}, 1 \leq i \leq m
\end{array}\right.
$$

$D_{n i}(z)$ represents the unit vector of $\mathrm{D}_{\mathrm{i}}$ within the boundaries of A. $\vec{D}_{i a v}$ denotes the mean value of A (as defined in (8). The aforementioned equation shows that the routing problem is decomposed into several optimization problems. For the $i^{\text {th }}$ destination node,

$$
\left\{\begin{array}{l}
\min \text { imize } J_{i}=\int_{A}\left|\left(\vec{D}_{i}-\vec{D}_{i a v}\right)\right|^{2} d s \\
\vec{\nabla} \cdot \vec{D}_{i}=\rho_{i} \\
D_{n i}(\mathrm{z})=0 \quad \mathrm{z} \in \text { the edge of area A }
\end{array} .\right.
$$

or

$$
\left\{\begin{array}{l}
\vec{\nabla} \cdot \bar{D}_{i}=\rho_{i} \\
\vec{\nabla} \times \bar{D}_{i}=0 \\
D_{n i}(\mathrm{z})=0 \mathrm{z} \in \text { the edge of area } \mathrm{A}
\end{array} .\right.
$$

The function defined by (17) cannot generate a unified solving form for an optimum solution because the load vector fields of different destination nodes are mutually independent. In this case, the cost form is changed to realize the unified comprehensive optimum solution form. The following indicates a modified form:

$$
J=\int_{A}\left(\sum_{i=1}^{m}\left|\vec{D}_{i}-\vec{D}_{i a v}\right|\right)^{2} d s .
$$

The cost function is continuous but not quadratic except when $m=1$. When the cost function is defined as shown in (20), an equation satisfying this equation is difficult to determine; however, the problem can be solved with a mathematical method.

\section{SIMULATION AND ANALYSIS}

This section presents the experimental result of load balancing. Nodes are distributed in an area measuring 500 $\mathrm{m}^{2} \times 500 \mathrm{~m}^{2}$, and the number of routing nodes is 300 . The section simulates the experimental scenarios of multisource and single-sink networks as well as of multi-source and multi-sink networks. In the former situation (Fig. 4), four source nodes are distributed in the four corners of the deployment area while the sink node is in the center of the area; in the latter scenario, the positions of source nodes remain unchanged while four new sink nodes are added (Fig. 5).

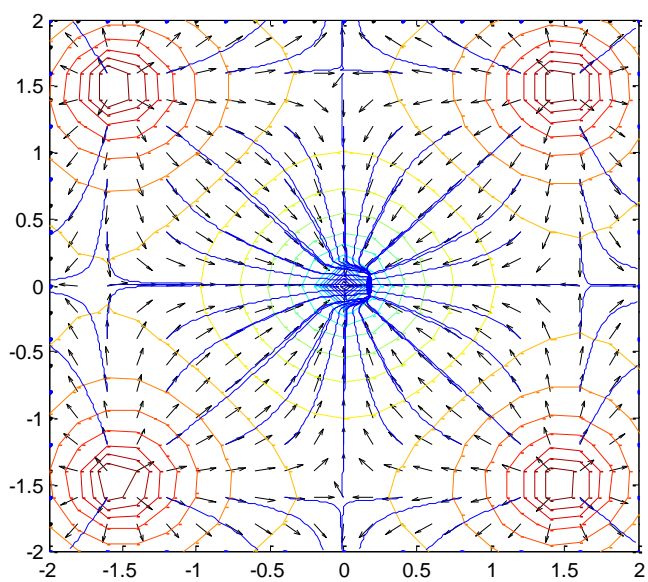

Figure 4. Distribution of Nodes in Multi-source and Single-sink Scenario

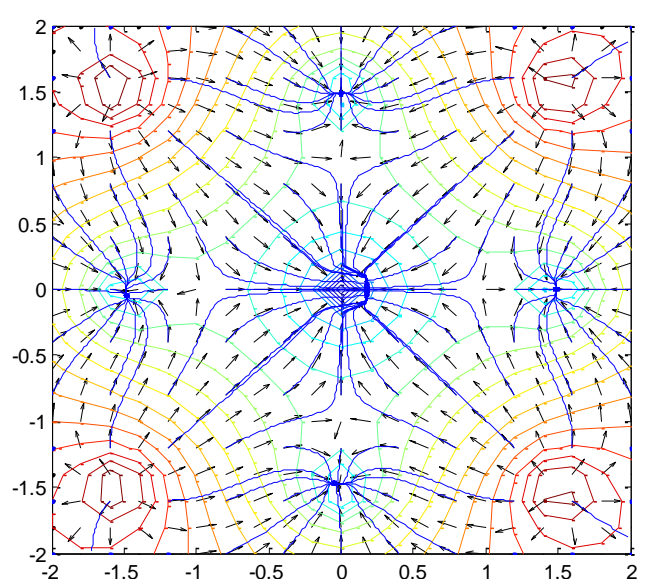

Figure 5. Distribution of Nodes in Multi-source and Multi-sink Scenario 
Fig. 4 and Fig. 5 illustrate the direction of $\vec{D}$ in the case of given source nodes, and the central node $(0,0)$ represents the destination node. The boundary conditions are divided as defined by (16) into $20 \times 20$ grids, and then the Poisson's equation is solved as per (14) with numerical values to identify the potential function U. Subsequently, $\vec{D}$ is obtained with this function. In the fig. 5, the line segment indicates the direction of optimal $\vec{D}$; this variable diverges in the source node and converges in the destination node. Fig. 4 indicates that $\vec{D}$ deviates in and approaches the destination node.

In the experiment, information is issued evenly to multiple paths according to the need of each node, i.e., information is transmitted through many paths from the source node to the destination node and the loading capacity of each path is related to the value of $\vec{D}$. Only the source node can issue information, which flows to the destination node in the direction of optimal $\vec{D}$ once the information is split. Fig. 6 and Fig. 7 depict the two scenarios based on the method in which $\circ$ represents the source node position and $\Delta$ denotes the sink node position. Each source node has multiple routes to the nearest sink node.

This study uses the Matlab platform for routing simulation and analysis in addition to adopting the following four indices to evaluate the performance of routing protocols: network delay, throughput, successful delivery rate, and energy consumption.

This work compares the proposed vector routing protocol (electrostatic theory routing, ET) with three classical multi-path routing protocols: ad-hoc on-demand distance vector (AODV), flooded forward ant routing $(\mathrm{FF})$, and sensor-driven and cost-aware ant routing (SC). Table 1 lists the simulation parameters.

TABLE I. SimULATION PARAMETERS

\begin{tabular}{|l|l|}
\hline \multicolumn{1}{|c|}{ Parameter } & \multicolumn{1}{c|}{ Value } \\
\hline Routing Protocol & ET, AODV, FF, SC \\
\hline Number of Nodes & 300 \\
\hline Maximum Hop Count & 20 \\
\hline Data Transmission & Constant Bit Rate (CBR) \\
\hline Transmission Rate & $300 \mathrm{kbps}$ \\
\hline Simulation Time & $100 \mathrm{~s}$ \\
\hline Node Energy & $50 \mathrm{~J}$ \\
\hline
\end{tabular}

In Fig. 8, the network delay of ET is much lower than that of FF but is slightly higher than those of SC and AODV because ET establishes multiple paths from the source node to the destination node, including the shortest path. The average hop count of all paths is higher than that of the shortest path; thus, the network delay is slightly higher. The simulation result shows that the network delay of ET is $0.0454 \mathrm{~s}$, whereas that of AODV is $0.0380 \mathrm{~s}$, in which case the former is approximately $25 \%$ higher than the latter.

Fig. 9 indicates that the throughput of ET is higher than those of the other three routing protocols because ET can transmit data in multiple paths simultaneously, whereas AODV has only one transmission path. The simulation result suggests that 512 data packets are sent in AODV, whereas 1,485 data packets (approximately thrice that of the aforementioned protocol) are sent in ET. Moreover, the throughputs of SC and FF tend to increase over time; by contrast, ET does not display this tendency and its throughput remains stable at the level of roughly 3.8.

Fig. 10 suggests that the successful delivery rate of ET in the entire simulation process is higher than $90 \%$, which indicates that more data packets can reach the destination node correctly. This rate has approached $91.5 \%$ since the 30th second. By contrast, the successful delivery rates of the other three protocols were very low at the beginning of the simulation process. In addition, the indices for SC and FF increase, whereas that for AODV decreases. Therefore, the successful delivery rate of ET is much higher than those of the other three protocols (the highest point is approximately $50 \%$ higher than the lowest point).

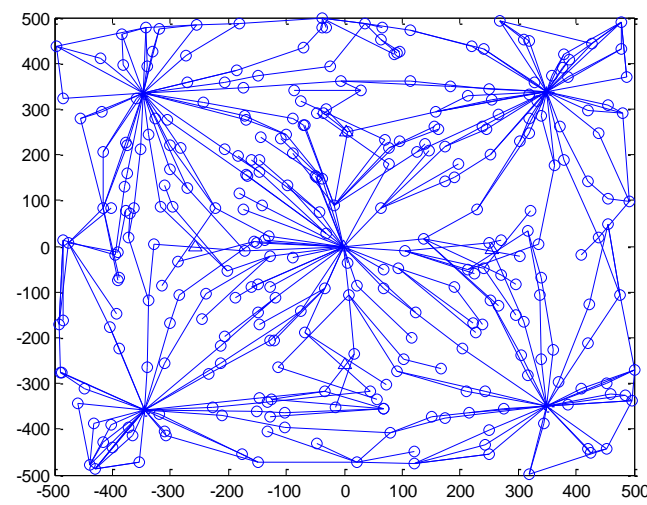

Figure 6. Simulation Diagram in Multiple-source and Single-sink Scenario

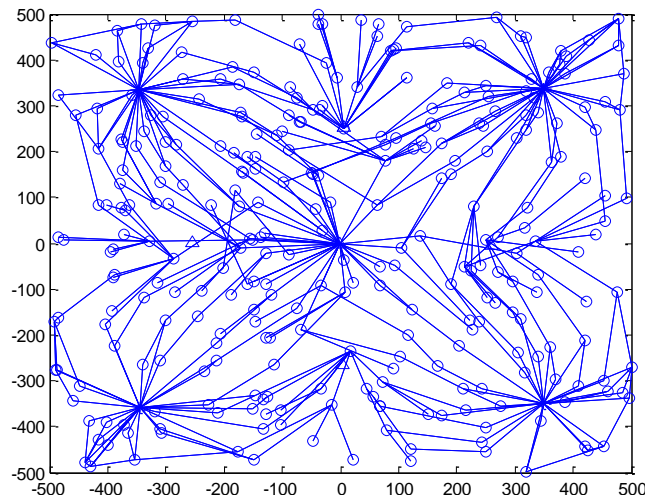

Figure 7. Simulation Diagram in Multiple-source and Multiple-sink Scenario

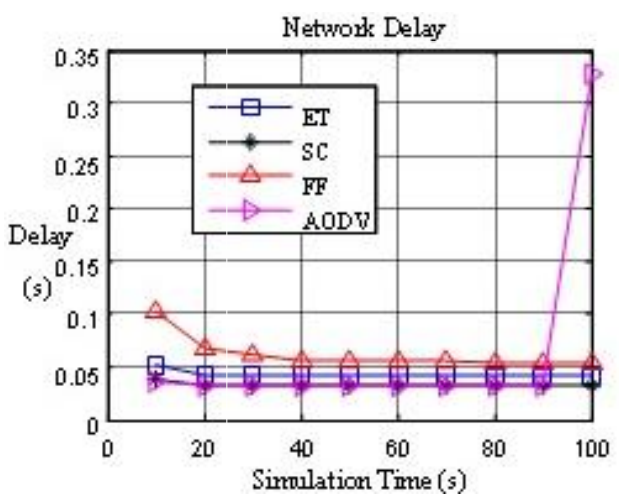

Figure 8. Network Delay 
Fig. 11 shows that network energy consumption increases with the progression of simulation in all protocols. AODV consumes the most energy, whereas SC consumes the least. Moreover, the curve slope of AODV is higher than that of ET, thereby indicating that the incremental energy consumption of the former rises over time.

\section{CONCLUSIONS}

This research constructs a multipath algorithm for WSN given two scenarios, i.e., the multi-source and single-sink situation and the multi-source and multi-sink scenario. Based on a comparison with the traditional routing algorithm, the conclusion is that network load distribution is balanced and throughput increases when the node position and the bandwidth demand of the source node are known. The next step is to consider the mobility of the source node; in the case of source node flow, the vector field will change over time, as will the routing.

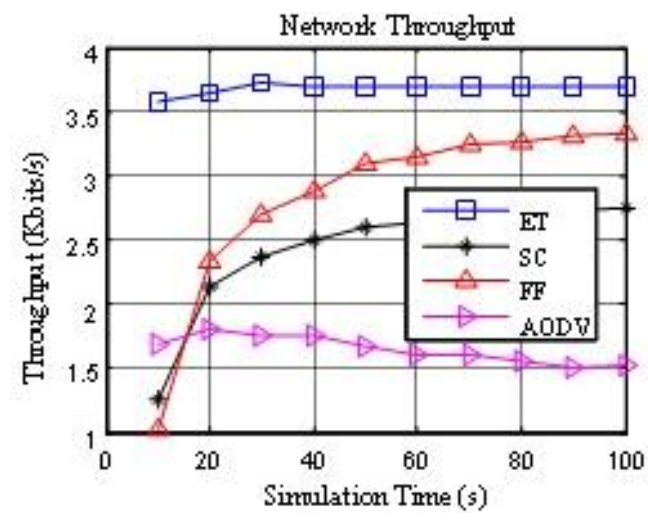

Figure 9. Network Throughput

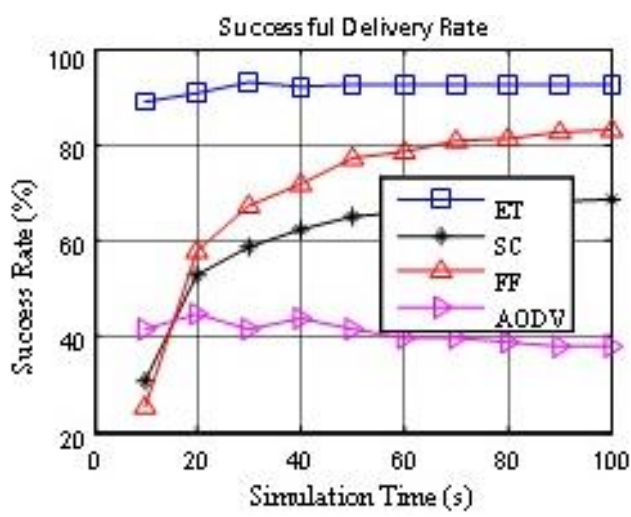

Figure 10. Successful Delivery Rate

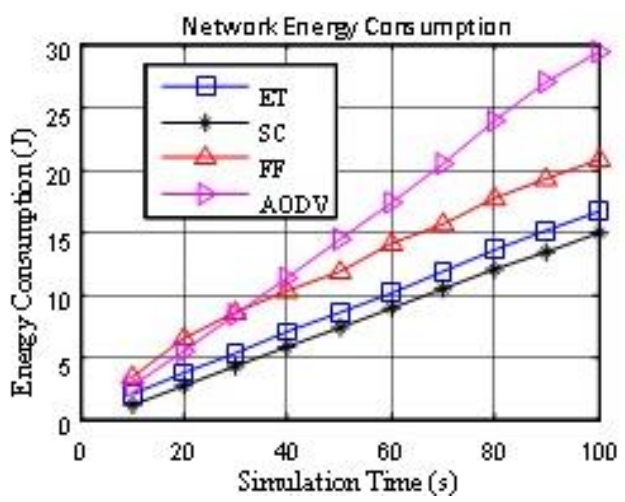

Figure 11. Network Energy Consumption

\section{REFERENCES}

[1] Feng Qu, Hua Yang, Lijun Wang, "The Wireless Sensor Network and Application", Journal of Sichuan Ordnance, vol. 34, no.2, pp.111-113, 2013.

[2] Xinghong Shang, "The Research on Key Technologies of Wireless Sensor Network", Doctoral Dissertation of Nanjing University of Science and Technology, China, 2013.

[3] Wei Zhao, "The Multipath Routing Protocol of Wireless Sensor Network Based on Vector Field and Network Coding", Nanjing University of Science and Technology, doctoral dissertation, 2014.

[4] Liu Jun, Song Guoping, "Development of Study on Information Process of Wireless Sensor Network", Logistics Technology, vol. 32, no.9, pp. 405-408, 2013.

[5] Jiang H F, Qian J S, Sun Y J, "Virtual Electrostatic Field Based Multi-sink Routing Algorithm in Wireless Sensor Network", Journal of China University of Mining \& Technology, vol. 40, no. 2, pp.321-326, 2011.

[6] Ming Z, Wang H, Xu M, "Evaluation of Path Stretch in Scalable Routing System", International Journal of Machine Learning \& Cybernetics, vol. 6, no. 2, pp.1-7, 2014.

[7] Chellathurai A S, Raj E G D P, "Evolutionary Fisheye State Routing Protocol in Mobile Adhoc Networks", International Journal of Computer Trends \& Technology, vol.14, no. 2, pp.100113, 2014. http://dx.doi.org/10.14445/22312803/IJCTT-V14P118

[8] Ahlawat S, Malik S, Goyal D, "Adhoc on Demand Distance Vector Routing Protocol (AODV): A Review Study", International Journal of Computer Science \& Management Studies, vol.10, no. 6, pp.309-321, 2013.

[9] Kalantari, Mehdi; Shayman, Mark, "Routing in Wireless ad hoc Networks by Analogy to Electrostatic Theory", IEEE International Conference on Communications, vol.7, Paris, June 20-24, 2004, pp.4028-4033. http://dx.doi.org/10.1109/icc.2004.1313307

[10] Dexiang Zhou, "The Research on Routing Protocol of Wireless Sensor Network", Doctoral Dissertation of Nanjing University of Posts and Telecommunications, China, 2013.

[11] Singh, Ruby; Kant, Shashi; Khatri, Pallavi, "Residual Energy Based Multipath Routing Protocol in Wireless Sensor Network", International Conference on Computing, Communication and $\mathrm{Au}-$ tomation, Greater Noida, May 15-16, 2015, pp. 420-426. http://dx.doi.org/10.1109/ccaa.2015.7148412

[12] Biswas U, Sarddar D, "Electrostatic Field Based Reliable Routing in Wireless Sensor Networks Using Vector Method", International Journal of Advances in Engineering \& Technology, vol.6, no.1, pp.296-308, 2013.

[13] Yuwang Yang, Chunshan Zhong,Yamin Sun, Jingyu Yang, "Network Coding Based Reliable Disjoint and Braided Multipath Routing for Sensor Networks", Journal of Network and Computer Applications, vol.33, no. 4, pp.422-432, 2010. http://dx.doi.org/ 10.1016/j.jnca.2010.02.003

[14] Liping Lu, Fei Huang, Hong Zhang, Jian Zhao, Yuwang Yang, "Energy Analysis of the Sensor Networks with Several Paths Routing Model Based on Network Coding", Journal of Nanjing University of Science and Technology, vol.34, no. 4, pp. 436-440, 2010.

[15] Toumpis, Stavros; Tassiulas, Leandros, “Optimal Deployment of Large Wireless Sensor Networks", IEEE Transactions on Information Theory, vol.52, pp. 2935-2953, 2006. http://dx.doi.org/ 10.1109/TIT.2006.876256

[16] Wei ZHAO, Zhenmin TANG, Yuwang Yang, Jiming YU, Lei WANG, "Routing in Wireless Sensor Network based on Field Theory", Journal of Computational Information Systems, vol.8, no. 5, pp.1-8, 2012.

\section{AUTHORS}

Ming Li is with the School of Computer Science and Engineering, Nanjing University of Science and Technology, Nanjing, 210094, China (e-mail: 1m@ hhu.edu.cn).

HuanYan Qian is with the School of Computer Science and Engineering, Nanjing University of Science and Technology, Nanjing, 210094, China (e-mail: hyqian@mail.njust.edu.cn).

Submitted, December, 1, 2015. Published as resubmitted by the authors on December, 26, 2015. 